\title{
CARACTERIZAÇÃO DAS MADEIRAS DENOMINADAS DE PAU-DE-ESCORA COMERCIALIZADAS NA CIDADE DE MANAUS, AMAZONAS
}

\author{
Jonnys Paz Castro ${ }^{1}$, Francisco Tarcísio Moraes $\mathrm{Mady}^{2}$, Niro Higuchi ${ }^{3}$, \\ Francisco José de Vasconcellos ${ }^{4}$, Jorge Alves de Freitas ${ }^{5}$, Blenda Naara Santos da Silva ${ }^{6}$
}

(recebido: 22 de novembro de 2010; aceito: 28 de abril de 2012)

\begin{abstract}
RESUMO: Neste trabalho, objetivou-se caracterizar as madeiras comercializadas como pau-de-escora na cidade de Manaus, Amazonas. Esse produto é utilizado como apoio na construção de lajes. Com exceção dos grandes prédios de apartamentos, a maioria das construções e reformas residenciais ainda utiliza esse produto, inclusive em prédios públicos. As estimativas quantitativas de dimensão e as análises anatômicas macroscópicas foram realizadas em 450 "varas" de pau-de-escora apreendidas pelo Batalhão Ambiental-AM. Houve, ainda, aplicação de questionário junto aos estabelecimentos que as vendem, para avaliar a dinâmica do comércio deste produto. O comprimento médio encontrado foi de 3,74 $\pm 0,08 \mathrm{~m}$ (IC 95\%) e o diâmetro médio foi de 4,48 $\pm 0,03 \mathrm{~cm}$ (IC 95\%). As sete famílias mais abundantes encontradas foram: Fabaceae, Annonaceae, Sapotaceae, Lecythidaceae, Lauraceae, Burseraceae e Chrysobalanaceae. O preço médio geral encontrado em Manaus para a compra de pau-de-escora foi $\mathrm{R} \$ 2,63$ e o de venda foi de $\mathrm{R} \$ 3,94$. Em geral, o pau-de-escora consumido em Manaus é ilegal, da produção à comercialização. Há uma padronização quanto às dimensões nas quais esse produto é comercializado, assim como o preço, nas casas comerciais que vendem irregularmente o referido material.
\end{abstract}

Palavras-chave: Escoramento, mensuração, identificação anatômica de madeiras, atividade comercial.

\section{CHARACTERIZATION OF WOODS KNOWN AS PAU-DE-ESCORA MARKETED IN THE CITY OF MANAUS, AMAZONAS}

\begin{abstract}
The objective was to characterize the wood product commercially known as pau-de-escora in Manaus, Amazonas. Paude-escora is a key foundation material to build concrete lining or storey in tower buildings. The use of pau-de-escora is very popular in Manaus even in public building construction. To carry out the analyses and the anatomical identification of the material, we used 450 pau-de-escora poles, which were apprehended by the Environmental Battalion of Amazonas Police Force. It was also carried out a social survey to better understand the dynamics of the trade of this product in Manaus. The length of the 450 poles averaged 3.74 $\pm 0.08 \mathrm{~m}(\mathrm{CI} 95 \%)$ and diameter at the base averaged $4.48 \pm 0.03 \mathrm{~cm}(\mathrm{CI} 95 \%)$. These small differences related to the means indicate that the market requires a very clear dimension pattern for this wood product. The seven most abundant families encountered were: Fabaceae, Annonaceae, Sapotaceae, Lecythidaceae, Lauraceae, Burseraceae and Chrysobalanaceae. The selling price of pau-deescora pole in Manaus averaged $R \$ 2.63$, and the buying price averaged $R \$ 3.94$. The custody chain of pau-de-escora is completely illegal not only in Brazil, perhaps worldwide because it is part of the natural regeneration of primary or recovery forests.
\end{abstract}

Key words: Concrete lining, wood identification anatomy, commercial activity.

\section{INTRODUÇÃO}

Ao longo da história, o homem utilizou-se dos recursos das florestas para que pudesse sobreviver e evoluir. Nas florestas, encontramos uma matéria-prima que foi fundamental na história, a madeira. As civilizações antigas reconheceram sua dívida em relaçãoà madeira (PERLIN, 1992).
Na construção civil, a madeira é utilizada de diversas formas em usos temporários, como: fôrmas para concreto, andaimes e escoramentos (FERREIRA, 2003). A madeira bruta ou roliça é empregada em forma de tronco, utilizada com mais frequência em construções provisórias, como escoramento (PFEIL, 1978).

\footnotetext{
${ }^{1}$ Engenheiro Florestal, Doutorando em Ciência e Tecnologia da Madeira - Universidade Federal de Lavras/UFLA - Departamento de Ciências Florestais - 37200-000 - Lavras, MG, Brasil - jonnys_33@ hotmail.com

${ }^{2}$ Engenheiro Florestal, Professor M.Sc. em Agronomia Tropical - Universidade Federal do Amazonas/UFAM - Departamento de Ciências Florestais - 69077-000 - Manaus, AM, Brasil - tarcisio@mandic.com.br

${ }^{3}$ Engenheiro Florestal, Pesquisador D.Sc. em Engenharia Florestal - Instituto Nacional de Pesquisa da Amazônia/INPA - Laboratório de Silvicultura Tropical - Cx P. 478 - 69011-970 - Manaus, AM, Brasil - niro@inpa.gov.br

${ }^{4}$ Especialista em Tecnologia de Madeira - Instituto Nacional de Pesquisas da Amazônia/INPA - Laboratório de Anatomia e Identificação de Madeiras - Cx P. 478 - 69060-000 - Manaus, AM, Brasil - chicao@inpa.gov.br

${ }^{5}$ Especialista em Tecnologia Florestal - Instituto Nacional de Pesquisas da Amazônia/INPA - Laboratório de Anatomia e Identificação de Madeiras - Cx P. 478 - 69060-000 - Manaus, AM, Brasil - jorginho@inpa.gov.br

${ }^{6}$ Engenheira Florestal, Mestranda em Agronomia/Fitopatologia - Universidade Federal de Lavras/UFLA - Departamento de Fitopatologia DFP/UFLA - 37200-000 - Lavras, MG, Brasil - blenda_g2@hotmail.com
}

Cerne, Lavras, v. 18, n. 4, p. 557-563, out./dez. 2012 
Madeira roliça é o produto com menor grau de processamento da madeira, que se usa para sustentar alguma coisa. Consiste de um segmento do fuste da árvore, obtido por cortes transversais (traçamento) ou mesmo sem esses cortes (varas: peças longas de pequeno diâmetro), empregados de forma temporária, em material de apoio na sustentação de lajes (FERREIRA, 2003).

As madeiras roliças, utilizadas na construção civil como elementos de sustentação de formas e lajes, são denominadas de pau-de-escora na cidade Manaus. Porém, em outros lugares recebem diferentes termos como escora de madeira, pontaletes, espeque e pauroliço.

As madeiras utilizadas como pau-de-escora, na cidade de Manaus, são árvores jovens retiradas da floresta, afetando a regeneração da floresta e desaparecimento de algumas espécies, causando impacto ambiental. Essa madeira não sofre nenhum processamento, sendo utilizada de forma rústica.

Essa matéria-prima, geralmente, é comercializada ilegalmente em lojas de materiais de construção, sendo vendidas por preço muito abaixo de seu valor real. Por se tratar de árvores jovens, são desconhecidas cientificamente as espécies, tampouco suas características. Sendo assim, torna-se relevante obter informações sobre as madeiras comercializadas como pau-de-escora em Manaus, Amazonas.

Nesse contexto, conduziu-se este trabalho com o objetivo geral de caracterizar as peças de madeira, denominadas de pau-de-escora, comercializadas na cidade de Manaus. Especificamente, pretende-se determinar as dimensões do pau-de-escora comercializado; realizar a identificação anatômica dessa madeira e verificar qual é o preço e a quantidade comercializada do pau-de-escora em Manaus.

\section{MATERIAL E MÉTODOS}

\section{1 Área de estudo e obtenção de pau-de-escora para} mensuração

O estudo foi realizado na cidade de Manaus, AM. Assinou-se o convênio entre o Batalhão Ambiental da Policia Militar do Amazonas (Batalhão Ambiental) e a Universidade Federal do Amazonas. No pátio do Batalhão, havia uma grande quantidade de paus-deescora que foi apreendida, os quais seriam comercializados na cidade de Manaus e que foram utilizados para a mensuração e identificação anatômica.

\subsection{Coletas de dados}

Foram realizadas três visitas no Batalhão de Policiamento Florestal, com o objetivo do referido trabalho. Os paus-de-escora foram escolhidos aleatoriamente do estoque do Batalhão, foram escolhidas 450 varas de paude-escora. O comprimento foi mensurado com fita métrica de $10 \mathrm{~m}$ e o diâmetro foi mensurado com paquímetro digital, de precisão $0,02 \mathrm{~mm}$, conforme a Figura 1 .

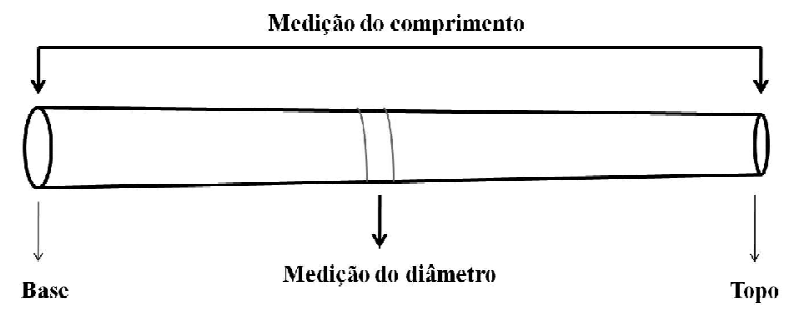

Figura 1 - Representação da medição do comprimento e diâmetro.

Figure 1-Representation oflength and diameter measurement.

Após a coleta dos dados de comprimento e diâmetro, estes foram organizados em planilha digital para posterior análise. Foram realizados, também, cálculos de estatística descritiva para ambas as variáveis, como: valor mínimo, valor máximo, média, desvio padrão, variância e erro padrão.

Para melhor conformidade, ao analisar os dados das medições, estes foram ordenados em ordem crescente. Depois, agrupados em classes, conforme a Tabela 1. O comprimento foi organizado em 11 classes com amplitude de $0,20 \mathrm{~m}$. O diâmetro foi organizado em 10 classes com uma amplitude de $0,6 \mathrm{~cm}$.

Tabela 1 - Distribuição do comprimento e diâmetro em classes.

Table 1 - Distribution of length and diameter classes.

\begin{tabular}{ccc}
\hline $\mathrm{N}$ & Classe de comprimento $(\mathrm{m})$ & Classe diamétrica $(\mathrm{cm})$ \\
\hline 1 & $2,71 \leq \mathrm{C}<2,91$ & $2,4 \leq \mathrm{d}<3,0$ \\
2 & $2,91 \leq \mathrm{C}<3,11$ & $3,0 \leq \mathrm{d}<3,6$ \\
3 & $3,11 \leq \mathrm{C}<3,31$ & $3,6 \leq \mathrm{d}<4,2$ \\
4 & $3,31 \leq \mathrm{C}<3,51$ & $4,2 \leq \mathrm{d}<4,8$ \\
5 & $3,51 \leq \mathrm{C}<3,71$ & $4,8 \leq \mathrm{d}<5,4$ \\
6 & $3,71 \leq \mathrm{C}<3,91$ & $5,4 \leq \mathrm{d}<6,0$ \\
7 & $3,91 \leq \mathrm{C}<4,11$ & $6,0 \leq \mathrm{d}<6,6$ \\
8 & $4,11 \leq \mathrm{C}<4,31$ & $6,6 \leq \mathrm{d}<7,2$ \\
9 & $4,31 \leq \mathrm{C}<4,51$ & $7,2 \leq \mathrm{d}<7,8$ \\
10 & $4,51 \leq \mathrm{C}<4,71$ & $\mathrm{~d} \geq 7,8$ \\
11 & $\mathrm{C} \geq 4,71$ & --- \\
\hline
\end{tabular}

Cerne, Lavras, v. 18, n. 4, p. 557-563, out./dez. 2012 


\subsection{Identificação do pau-de-escora}

A identificação anatômica macroscópica de madeiras foi realizada no laboratório de Física da Madeira, da Universidade Federal do Amazonas e no laboratório de Anatomia e Identificação de Madeiras/Xiloteca, da Coordenação de Pesquisas em Produtos Florestais do Instituto Nacional de Pesquisas da Amazônia, sendo o método adotado para o procedimento de identificação das amostras o de confronto por comparação, utilizando-se o uso de uma lupa do tipo conta-fio com aumento 10x, para uma melhor observação dos tecidos lenhosos das espécies.

Com as amostras de madeiras convenientemente preparadas para a análise macroscópica, foram feitas as observações baseadas na metodologia que é utilizada nos estudos de Anatomia de Madeiras e, associada a outras publicações cientificas, das quais citam-se: Comissión Panamericana de Normas Técnicas - COPANT (1974), Comitee on Nomenclature International Association of Wood Anatomists - IAWA Committee (1964), Freitas et al. (1992), Instituto Brasileiro do Meio Ambiente e dos Recursos Naturais Renováveis - IBAMA (1991), Loureiro et al. (1994) e Vasconcelos et al. (2001). Após a identificação anatômica, realizou-se a padronização quanto à classificação botânica segundo a Angiosperm Phylogeny Group - APG (2003).

\subsection{Levantamento sobre o comércio do pau-de-escora}

Para a realização do levantamento sobre o comércio de pau-de-escora, foram escolhidos, aleatoriamente, 5 estabelecimentos (lojas de materiais de construção) que revendiam essa matéria-prima, em cada zona administrativa da cidade (Centro-sul, Centro-oeste, Leste, Norte, Oeste e Sul), totalizando, assim, 30 estabelecimentos.

Para contemplar essa etapa da pesquisa foi elaborado um questionário para obter informações sobre quantidade comprada e vendida, preço de compra e venda por unidade (varas) junto aos estabelecimentos. Os dados foram analisados no software SISVAR, pelo delineamento inteiramente casualizado. Os parâmetros máximo e mínimo também foram incluídos.

\section{RESULTADO E DISCUSSÃO}

3.1 Caracterização das dimensões do pau-de-escora comercializado

Na Figura 2, ilustra-se a distribuição dos valores encontrados para o comprimento em classes, onde observa-se que a classe que obteve a maior frequência foi a classe $6(3,71 \leq \mathrm{C}<3,91)$.

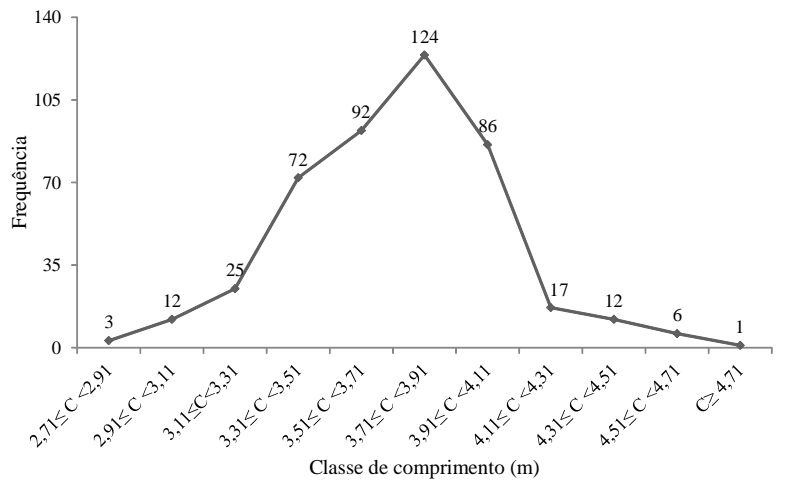

Figura 2 - Distribuição de comprimento observada por classe.

Figure 2 - Length distribution observed by class.

Nota-se, ainda, que há poucos indivíduos com comprimento abaixo de $3,30 \mathrm{~m}$ e superior a $4,11 \mathrm{~m}$, destacando que, abaixo de $2,91 \mathrm{~m}$, a freqüência representa $0,6 \%$ do total e acima de 4,71 representa apenas $0,2 \%$.

A partir da distribuição da classe diamétrica, Figura 3 , verificou-se que os diâmetros mais frequentes estão situados na classe $3(3,6 \leq d<4,2)$, seguido das classes $4 \mathrm{e}$ 5. Pode-se observar que entre 3,0 a $5,9 \mathrm{~cm}$, estão representados $93 \%$ dos indivíduos mensurados.

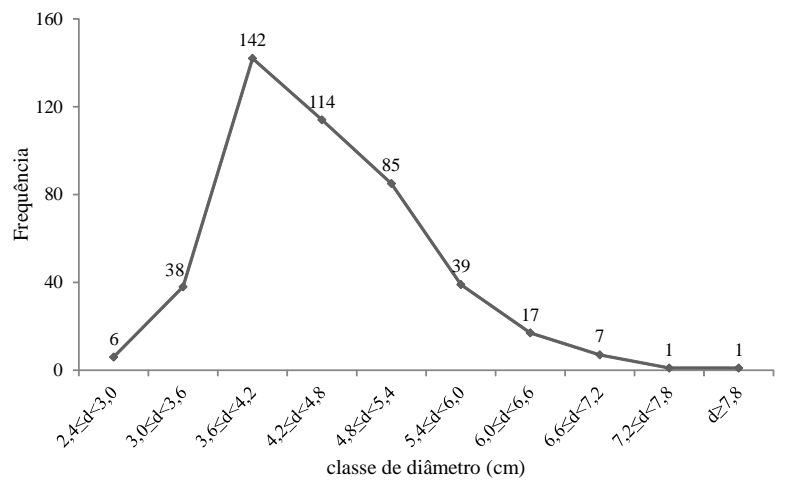

Figura 3 - Distribuição diamétrica observada por classe.

Figure 3 - Diameter distribution observed by class.

Entre 3,6 a 4,7 cm, estão representados $62 \%$ dos indivíduos, demonstrando, assim, que esse último intervalo corresponde à preferência comercial. Acima de 7,2cm, a frequência é diminuta, representando $2 \%$ dos indivíduos, e acima de $7,8 \mathrm{~cm}$ representa $0,2 \%$ dos indivíduos.

A mensuração dos paus-de-escora revelou um comprimento médio de $3,74 \pm 0,03 \mathrm{~m}$ (I.C. $95 \%$ )* e o diâmetro

Cerne, Lavras, v. 18, n. 4, p. 557-563, out./dez. 2012 
médio foi de $4,48 \pm 0,08 \mathrm{~cm}$ (I.C. $95 \%$ )*. Ou seja, para o comprimento a incerteza é de $0,03 \mathrm{~m}$ de uma média de $3,74 \mathrm{~m}$ isso corresponde a $0,8 \%$ de erro, enquanto para o diâmetro a incerteza é de $0,08 \mathrm{~cm}$ de uma média de 4,48 , correspondendo a $1,76 \%$ de erro. Em geral, nos inventários, as incertezas toleráveis são de 10 a $20 \%$, o que estabelece que os resultados encontrados sejam admissíveis. Foram apresentados, na Tabela 2, alguns dados de estatística descritiva para uma melhor análise e compreensão dos resultados.

Mesmo com média de 3,74 m, ainda assim, há uma perda na utilização do pau-de-escora em construções. A altura de um andar é, em média, de $3 \mathrm{~m}$, ou seja, para a utilização desse recurso para a sustentação de lajes, essas varas superiores devem ser novamente cortadas. Portanto, utilizando-se varas de pau-de-escora no comprimento médio encontrado, seria gerado $0,74 \mathrm{~m}$ de resíduo por vara, cerca de $20 \%$ de perda.

Não foi encontrado pau-de-escora superior a $10 \mathrm{~cm}$ de diâmetro. Isso se deve ao fato de segmentos de fuste com diâmetros inferiores a $10 \mathrm{~cm}$, juntamente com um comprimento não muito extenso, facilitar o manuseio e transporte no processo de retirada da floresta.

A legislação estadual, por meio da Instrução Normativa $n^{\circ} 005$, especifica que o Diâmetro Mínimo de Colheita (DMC) é de $50 \mathrm{~cm}$ para todas as espécies. Portanto, a retirada com diâmetro muito abaixo do recomendado estabelece em um ato ilegal do comércio de pau-de-escora. "Art. $7^{\circ}$. Fica estabelecido o Diâmetro Mínimo Colheita de 50 com para todas as espécies para as quais ainda não estabeleceu o DMC específico" (AMAZONAS, 2008).

Além disso, esse recurso florestal é explorado e comercializado sem apresentação do Documento de Origem Florestal (DOF), o que implica no primeiro crime ambiental, na retirada de pau-de-escora para ser comercializado.

\subsection{Madeiras comercializadas como pau-de-escora}

Na identificação anatômica macroscópica, foram identificadas 31 famílias, 61 gêneros, 13 espécies e 37 amostras não identificadas. Em alguns casos foram identificadas apenas ao nível de família, portanto, não sendo possível identificar o gênero e a espécie.

As famílias mais importantes, em ordem decrescente no número de indivíduos, foram: Fabaceae (83), Annonaceae (43), Sapotaceae (36), Lecythidaceae (33), Lauraceae (29), Burseraceae (27) e Chrysobalanaceae (21). Juntas, essas sete famílias responderam por quase $60,44 \%$ dos indivíduos identificados, ficando as 24 famílias restantes, responsáveis por $31,33 \%$ do total, evidenciando a baixa frequência relativa de indivíduos nessas famílias, menor que 20 indivíduos por família, e não identificadas $8,22 \%$, Figura 4.

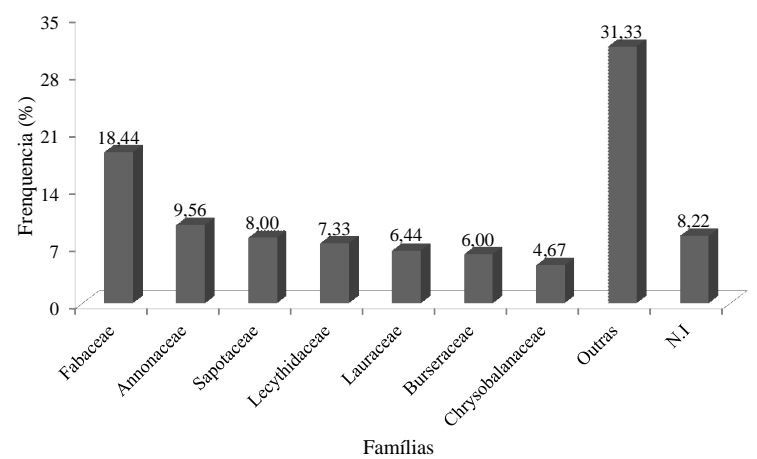

Figura 4 - Distribuição percentual e comparação das famílias identificadas.

Figure 4 - Percentage distribution and comparison of the identified families.

Em estudo diagnóstico da regeneração natural, Higuchi et al. (1985), em uma floresta na área do Distrito Agropecuário da SUFRAMA, em relação ao número de indivíduos com $\mathrm{DAP} \leq 5$, destacou-se a presença de espécies das famílias de Lauraceae em $1^{\circ}$ lugar, Lecythidaceae em $2^{\circ}$ lugar e Myristicaceae em $3^{\circ}$ lugar.

Em estudo florístico e fitossociológico, realizado por Oliveira e Amaral (2004) com árvores DAP $\geq 10 \mathrm{~cm}$, em

Tabela 2 - Estatística descritiva e erros do comprimento e diâmetro do pau-de-escora ( $\mathrm{N}=450)$.

Table 2 - Descriptive statistics and errors in length and diameter of pau-de-escora $(N=450)$.

\begin{tabular}{lcccccccc}
\hline Variáveis & Mínimo & Média & Máximo & Variância & Desvio Padrão & C.V (\%) & Erro Pad. da Média $^{1}$ & Erro Pad. da Amost. $^{2}$ \\
\hline Comprimento (m) & 2,73 & 3,74 & 5,00 & 0,10 & $\pm 0,32$ & 8,63 & $\pm 0,02$ & $\pm 0,03$ \\
Diâmetro (cm) & 2,40 & 4,48 & 8,80 & 0,72 & $\pm 0,85$ & 19,00 & $\pm 0,04$ & $\pm 0,08$ \\
\hline
\end{tabular}

*(I.C. $)=$ Intervalo de confiança com $95 \%$ de probabilidade; $\mathrm{N}=$ número de amostras.

$\mathrm{C} . \mathrm{V}(\%)=$ coeficiente de variação; ${ }^{1}$ - erro padrão de média; ${ }^{2}$ - erro padrão da amostragem.

Cerne, Lavras, v. 18, n. 4, p. 557-563, out./dez. 2012 
uma floresta de vertente na Amazônia Central encontraram em ordem decrescente no número de indivíduos, as famílias mais importantes: Lecythidaceae (118), Burseraceae (87), Sapotaceae (78), Chrysobalanaceae (72), Euphorbiaceae (62) e Fabaceae (43).

As varas de pau-de-escora são retiradas, geralmente, de um local vulgarmente denominado de 'varal', zona de regeneração florestal, marcado pela abundância de árvores jovens, onde são cortadas todas as espécies dentro do tamanho padrão, inclusive as madeiras de lei (KURIHARA; CARDOSO, 2009).

Assim, sem informações específicas das espécies encontradas na regeneração natural é inapropriado fazer uma comparação das famílias na identificação do pau-deescora com as famílias em inventário florestal. Talvez, essa diferença ocorra, em decorrência do fato de que as informações de inventários sejam de indivíduos com $\mathrm{DAP} \geq 10 \mathrm{~cm}$ e os indivíduos de pau-de-escora estarem abaixo desse diâmetro.

Em relação aos gêneros, os 12 mais frequentes, em ordem decrescente, foram Vismia sp.(19), Licania sp. (14), Escheweilera sp. (11), Inga sp. (10), Protium sp.(8), Annona sp (7), Virola sp (7), Parkia sp. (6), Swartizia sp. (6), Ocotea sp (5), Onychopetalum sp. (5) e Ormosia sp (5). Os demais gêneros tiveram frequência menor que 5 indivíduos.

Também foram registrados gêneros importantes de espécies comerciais como Couratari sp., Hymenolobium sp., Peltogyne sp., Vatairea sp. e Minquartia sp. Destacase, ainda, a presença do gênero Copaifera $\mathrm{sp}$.

Conforme o Decreto N. ${ }^{\circ} 25.044$, de $1 .^{\circ}$ de junho de 2005, do Estado do Amazonas, que proíbe o licenciamento do corte, transporte e comercialização de madeira das espécies de andirobas e copaíbas, percebe-se, com isso, o desrespeito à Política Nacional de Meio Ambiente e, posteriormente, à legislação de proteção ambiental. "Art. $1 .^{\circ}$ Fica proibido o licenciamento do corte, transporte e comercialização da madeira de andiroba "Carapa guianensis", "Carapa paraense" e copaibeira "Copaifera trapezifolia Hayne","Copaífera reticulata" e "Copaifera multijuga" (AMAZONAS, 2005).

Nota-se que, além de serem retiradas árvores, com diâmetro abaixo do estabelecido pela legislação, o comércio do pau-de-escora comete mais um crime ambiental, em razão da presença de espécies do gênero Copaifera sp. e outras madeiras proibida de corte.

As 13 espécies identificadas foram: Vismia guianensis (Aubl.) Choisy; Ormosia paraensis Ducke; Euxylophora paraensis Hub.; Sacoglottis guianensis
Benth; Calycophyllum spruceanum (Benth) K. Schum, Parahancornia amapá (Huber) Ducke; Peltogyne catingae Ducke; Couma macrocarpa (Barb.) Rodr.; Schefflera morototoni (Aubl.) Frondin; Pradosia praealta (Ducke) T.D. Penn; Himatanthus sucuuba (Spruce) Woodson; Amphirrhox surinamensis Eichler; e Duckeodendron cestroides Kuhlm.

\subsection{Atividade comercial do pau-de-escora}

Nota-se que a retirada de pau-de-escora, com a finalidade de utilização para a construção civil, ocorre dentro do perímetro de Manaus e na região metropolitana da capital do estado.

Na região do rio Cueiras, margem esquerda do rio Negro, no Amazonas, próximo a cidade de Manaus, nas comunidades de povos tradicionais e de ribeirinhos, é comum a passagem dos "vareiros", pessoas que comercializam o pau-de-escora, que utilizam os barcos regionais, singrando o rio, comprando as varas dos moradores ou trocando por produtos da cidade, num sistema similar ao do aviamento. $O$ preço pago a cada vara geralmente varia entre $\mathrm{R} \$ 0,40$ a $\mathrm{R} \$ 0,60$, exigindo trabalho diário e carga horária de cerca de 8 horas, para obterem rendimento aceitável (KURIHARA; CARDOSO, 2009).

Houve uma dificuldade em obter essas informações referentes aos preços que pau-de-escora é revendido, durante a etapa de aplicação do questionário, em razão do receio dos proprietários ou gerentes das lojas de materiais de construção imaginarem ser um ato de algum órgão de fiscalização ambiental, mesmo tendo antes sido informados e, com as explicações necessárias, para a obtenção das respostas no questionário apresentado.

O preço médio geral, encontrado em Manaus, para a compra de pau-de-escora foi $\mathrm{R} \$ 2,63$ e o de venda de $\mathrm{R} \$ 3,94$. Não houve diferença estatística entre as médias para o preço de compra e venda nas 6 zonas da cidade, Tabela 3 .

$\mathrm{O}$ valor mínimo em que as lojas de materiais de construção compram esse produto é de R \$ 2,00 (Zonas Centro-oeste, Leste e Oeste) e o máximo é de R \$ 3,50 (zona centro Sul).

Para o preço de venda o mínimo foi de R\$ 3,50 e, registrado em todas as zonas e o preço máximo foi de $\mathrm{R} \$ 4,80$ (Zona Sul).

A quantidade de pau-de-escora comercializada encontrada, por mês, nas zonas da cidade, foi em média 717 varas na cidade de Manaus. Também não houve diferença estatística para a quantidade comercializada.

Segundo os "donos/gerentes" dos estabelecimentos, essa quantidade é uma estimativa

Cerne, Lavras, v. 18, n. 4, p. 557-563, out./dez. 2012 
Tabela 3 - Preços de compra e venda nas zonas da cidade.

Table 3 - Purchase and sale prices in the areas of the city.

\begin{tabular}{lcccccc}
\hline \multirow{2}{*}{ Zonas } & \multicolumn{3}{c}{ Preço de Compra (R\$) } & \multicolumn{3}{c}{ Preço de Venda (R\$) } \\
\cline { 2 - 7 } & Média & Mínimo & Máximo & Média & Mínimo & Máximo \\
\hline Centro-oeste & $2,48 \mathrm{a}$ & 2,00 & 2,70 & $3,88 \mathrm{a}$ & 3,50 & 4,50 \\
Centro-sul & $2,78 \mathrm{a}$ & 2,50 & 3,20 & $3,92 \mathrm{a}$ & 3,50 & 4,50 \\
Leste & $2,60 \mathrm{a}$ & 2,00 & 3,00 & $4,10 \mathrm{a}$ & 3,50 & 4,80 \\
Norte & $2,64 \mathrm{a}$ & 2,50 & 3,00 & $3,86 \mathrm{a}$ & 3,50 & 4,60 \\
Oeste & $2,50 \mathrm{a}$ & 2,00 & 3,00 & $3,82 \mathrm{a}$ & 3,50 & 4,50 \\
Sul & $2,80 \mathrm{a}$ & 2,50 & 3,50 & $4,06 \mathrm{a}$ & 3,50 & 4,80 \\
\hline Média & 2,63 & - & - & 3,94 & - & - \\
C.V. $(\%)$ & 13,22 & - & - & 11,85 & - & - \\
\hline
\end{tabular}

Médias seguidas da mesma letra não diferem entre si pelo teste Tukey ao nível de 5\% probabilidade.

C.V. (\%): coeficiente de variação.

grosseira e nem sempre é possível vender pau-de-escora em todos os meses do ano. Analisando a quantidade por zonas, na zona leste, foi obtido o maior índice médio de pau-de-escora comercializado, seguido pela zona Norte e Centro-oeste.

Na zona leste, em um dos estabelecimentos, houve o registro de uma quantidade máxima de 1945 varas comercializadas de pau-de-escora e, na zona sul, obteve-se a mínima quantidade, com 300 varas comercializadas, Tabela 4.

Tabela 4 - Quantidade comercializada de pau-de-escora.

Table 4-Marketed quantity of pau-de-escora.

\begin{tabular}{lccc}
\hline \multirow{2}{*}{ Zonas } & \multicolumn{3}{c}{ Quantidade comercializada } \\
\cline { 2 - 4 } & Média & Mínimo & Máximo \\
\hline Centro-oeste & $744 \mathrm{a}$ & 500 & 900 \\
Centro-sul & $700 \mathrm{a}$ & 550 & 980 \\
Leste & $829 \mathrm{a}$ & 300 & 1945 \\
Norte & $774 \mathrm{a}$ & 400 & 1050 \\
Oeste & $677 \mathrm{a}$ & 420 & 1200 \\
Sul & $580 \mathrm{a}$ & 300 & 800 \\
\hline Média & 717 & - & - \\
C.V. $(\%)$ & 46,72 & - & - \\
\hline
\end{tabular}

Médias seguidas da mesma letra não diferem entre si pelo teste Tukey ao nível de $5 \%$ probabilidade.

C.V.(\%): coeficiente de variação.

Considerando a média mensal de venda (717), são abatidas, anualmente, mais de 8 mil árvores nativas com diâmetro abaixo de $10 \mathrm{~cm}$, comercializadas na forma de madeira roliça, para sustentar a construção civil na cidade de Manaus, o que requer esforços específicos para normatizar essa atividade ilegal.

\section{CONCLUSÕES}

As madeiras retiradas para a comercialização como pau-de-escora possuem como principal características uma padronização para as dimensões.

As madeiras comercializadas, como pau-de-escora, possuem uma alta diversidade, em alguns casos, não sendo possível identificar a espécie, sendo a família Fabaceae com maior frequência dos indivíduos identificados.

Os preços de compra e venda dessa matéria-prima não diferem estatisticamente, assim como a quantidade comercializada.

\section{REFERÊNCIAS}

AMAZONAS. Secretaria do Meio Ambiente e Desenvolvimento Sustentável. Decreto $\mathbf{N}^{\circ} \mathbf{2 5 . 0 4 4}$, de 1 de junho de 2005. Proíbe o licenciamento do corte, transporte e comercialização de madeira das espécies de andirobeiras e copaibeiras. Manaus, 2 jun. 2005.

AMAZONAS. Secretaria do Meio Ambiente e Desenvolvimento Sustentável. Instrução Normativa $\mathbf{N}^{\mathbf{0}} \mathbf{0 0 5}$, de 26 de fevereiro de 2008. Dispõe sobre o procedimento técnico para elaboração, apresentação, execução e avaliação técnica do Plano de Manejo Florestal Sustentável-PMFS nas Florestas Nativas e formações sucessoras. Manaus, 26 fev. 2008.

Cerne, Lavras, v. 18, n. 4, p. 557-563, out./dez. 2012 
ANGIOSPERM PHYLOGENY GROUP. An update of the Angiosperm Phylogeny Group classification for the orders and families of flowering plants: APG II. Botanical Journal of the Linnean Society, London, v. 141, n. 4, p. 399-436, 2003.

COMISSÍON PANAMERICANA DE NORMAS

TÉCNICAS. Descripcion de características generales, macroscópicas de las maderas angiospermas dicotiledoneas. Caracas, 1974. 19 p.

COMITEE ON NOMENCLATURE INTERNATIONAL ASSOCIATION OF WOOD ANATOMISTS. Multilingual glossary of terms used in wood anatomy. Konkordia, 1964. $186 \mathrm{p}$.

FERREIRA, O. P. (Coord.). Madeira: uso sustentável na construção civil. São Paulo: Instituto de Pesquisas Tecnológicas, 2003. 58 p.

FREITAS, J. A.; VASCONCELLOS, F. J.; SILVA, N. B.; LOUREIRO, A. A. Madeiras da Amazônia que apresentam raios largos. Acta Amazonica, Manaus, v. 22, n. 1, p. 91-161, 1992.

HIGUCHI, N. JARDIM, F. C. S. SANTOS. J. dos. ALENCAR, J. da C. Inventário Diagnóstico da Regeneração Natural. Acta Amazônica, Manaus, v.15, n.1-2, p.1999-233, 1985.

INSTITUTO BRASILEIRO DO MEIO AMBIENTE E DOS RECURSOS NATURAIS RENOVÁVEIS. Normas de procedimento em estudos de anatomia da madeira: I.
Angispermae, II. Gimnospermae. Brasília: Laboratório de Produtos Florestais, 1991. 19 p. (Série técnica, 15).

KURIHARA, L. P.; CARDOSO, T. M. Exploração madeireira de pequena escala na Amazônia Central: uma ameaça aos sistemas agrícolas tradicionais? Revista Brasileira de Agroecologia, Santa Maria, v. 4, n. 2, p. 1314-317, 2009.

LOUREIRO, A. A.; FREITAS, J. A. de; SILVA, A. C. Chave para identificação macroscópica de 77 madeiras da Amazônia. Manaus: INPA/SEMACT/AM, 1994. 71 p.

OLIVEIRA, A. N.; AMARAL, I. L. Florística e fitossociologia de uma floresta de vertente na Amazônia Central, Amazonas, Brasil. Acta Amazônica, Manaus, v. 34, n. 1, p. 21-34, 2004.

PERLIN, J. História das florestas: a importância da madeira no desenvolvimento da civilização. Rio de Janeiro: IMAGO, 1992. $490 \mathrm{p}$.

PFEIL, W. Estruturas de madeira: dimensionamento segundo as normas brasileiras NBR11 e os modernos critérios das normas alemãs e americanas. Rio de Janeiro: LTC, 1978. $272 \mathrm{p}$.

VASCONCELLOS, F. J. de; FREITAS, J. A. de; LIMA, V. M. O. da C.; MONTEIRO, L. do V.; PEREIRA, S. de J.

Madeiras tropicais de uso industrial do Maranhão: características tecnológicas. Manaus: MCT/INPA/UFMA, 2001. 96 p. 